\title{
Backstepping Adaptive Fuzzy Control of Servo System With LuGre Friction
}

\author{
Haibo Zhao ${ }^{1,2, a}$, Chengguang Wang ${ }^{3}$ \\ ${ }^{1}$ Engineering Technology Research Center of Optoelectronic Appliance, Tongling University, Tongling Anhui, \\ 244061, China \\ ${ }^{2}$ Department of Electrical Engineering, Tongling University, Tongling Anhui, 244061, China \\ ${ }^{3}$ Sichuan Institute of Aerospace System Engineering, Chengdu Sichuan, 610100, China
}

\begin{abstract}
Aiming at the control problem of servo system with friction nonlinearity, we introduced the improved LuGre friction model of system. We used adaptive fuzzy system to approximate the nonlinear part and unknown parameters in system online. The purpose is to avoid the complex calculation in deducing the adaptive law of each unknown parameter. By using backstepping approach and recursively selecting the Lyapunov function, introducing the virtual control quantity, we designed an adaptive fuzzy controller with state feedback. Then we analyzed the stability of adaptive fuzzy controller. We carried out the system response analysis and system robustness analysis. We used sinusoidal signal as simulation input signal. Two cases were considered in simulation test comparing backstepping control with conventional PID control. Simulation results show that the proposed backstepping control has better position tracking performance and robustness than conventional PID control.
\end{abstract}

\section{Introduction}

In electromechanical servo system, the existence of friction nonlinearity will affect the system dynamic performance and steady-state precision [1]. Hence, it is necessary to study the control strategy of weakening the effect of friction nonlinearity to system. So far, scholars at home and abroad have carried on a large amount of researches in friction modeling. The famous models of friction nonlinearity including the Dahl model [2], the LuGre model[3], the Elasto-plastic model [4], the Leuven model [5] and the GMS model [6]. Among them, the LuGre model is widely used [7] because it is simple and accurate in describing the friction characteristics at low speed. However, when system is running in high speed, the system friction are mainly coulomb friction and viscous friction, if still using the LuGre model, it will inevitably increase the complexity of the system. In addition, due to the changes of temperature, lubrication and contact force, the parameters of friction torque will also change.

Many scholars have studied the compensation control strategies of friction nonlinearity. Y. Wang [8] proposed an effevtive observer-based compensation scheme for the effects of friction. The results show that the amplitudes of limit cycles are greatly reduced with the disturbance observer-based compensation, and the stick-slip motion at low velocities is also eliminated. S. Yang [9] proposed a new repetitive adaptive compensation scheme for the dynamic friction. Computer simulations verify

\footnotetext{
${ }^{a}$ Corresponding author : happyzhaohaibo@126.com
} 
the effectiveness of the proposed scheme for the high-precision servo system. In [10], aiming at unknown dynamic friction parameters and unknown load characteristic, an adaptive friction compensation is designed for the servo system. Simulation and experiment results are shown to effectively inhibit the disadvantageous influence of friction and lay the foundation for improvement of dynamic performance of servo system. In [11], a friction torque compensation controller without distal-end feedback is designed to ensure that the output of the tendon-sheath actuation system can effectively track the expected torque trajectory, and the effectiveness of the scheme is validated by experiments. B. Feng [12] proposed a novel adaptive configuration method of friction compensation pulse characteristic parameters. The experiment results show that the proposed method has a better performance. In [13], a feedforward friction observer is proposed as formulating an explicit analytical expression for the applied observation function. The simulation results show that the proportional control combined with the observer compensates faster for frictional disturbances at suddenly changing frictional conditions than PI control. M.T.S. Aung [14] presented a new method of friction compensation that can be applied to geared actuators with high presliding stiffness. The proposed method is validated through experiments employing a harmonic drive transmission. Howerer, [8-14] have not used adaptive fuzzy control.

Adaptive fuzzy logic system proposed by Wang [15] has universal approximation property, which can effectively approximate the nonlinear function of system. In recent years, it has been widely used in the nonlinear control of various servo systems [16-20]. In this paper, an improved LuGre model [21-22] is used to describe the friction torque of system at low speed and high speed. We use an adaptive fuzzy logic system to approximate the nonlinear part of servo system with friction, design a backstepping [23-24] adaptive fuzzy controller under the condition of the unknown system parameters and load torque. Simulation and practical experiments validate the efficacy of the proposed control strategy.

\section{System Modeling}

If only considering the influence of LuGre friction nonlinear, the structure diagram of servo system is shown in Fig. 1.

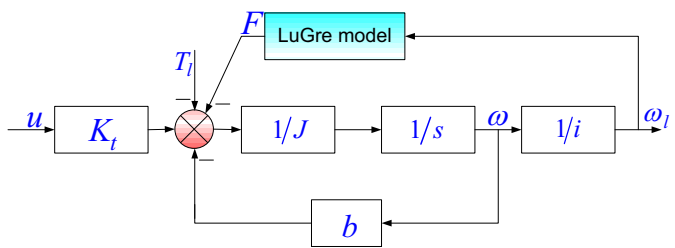

Figure 1. The structure diagram of servo system.

We can get the dynamic equation of servo system is as follows [1].

$$
\left\{\begin{array}{l}
\frac{d \theta}{d t}=\omega \\
J \frac{d \omega}{d t}=K_{t} u-b \omega-F-T_{l} \\
y=\theta
\end{array}\right.
$$

where $\theta$ and $\omega$ are the position and speed of motor output shaft respectively. $J$ and $b$ are equivalent moment of inertia and equivalent damping coefficient converted to the motor shaft respectively. $K_{t}$ is motor torque constant. $u$ is control variable. $F$ is load friction torque. $T_{l}$ is load torque converted to the motor shaft. If the LuGre model is adopted [21], then the load friction torque can be expressed as follows. 


$$
\begin{gathered}
\frac{d z}{d t}=s\left(\left|\omega_{l}\right|\right)\left(\omega_{l}-\frac{\left|\omega_{l}\right|}{g\left(\omega_{l}\right)} z\right) \\
F=\sigma_{0} s\left(\left|\omega_{l}\right|\right) z+\sigma_{1} \dot{z}+F_{c} \operatorname{sgn}\left(\omega_{l}\right)\left(1-s\left(\left|\omega_{l}\right|\right)\right)+\alpha_{2} \omega_{l} \\
\sigma_{0} g\left(\omega_{l}\right)=F_{c}+\left(F_{s}-F_{c}\right) e^{-\frac{\omega_{l}}{\omega_{s}}}=\alpha_{0}+\alpha_{1} e^{-\frac{\omega_{l}}{\omega_{s}}}
\end{gathered}
$$

Where

$$
s\left(\left|\omega_{l}\right|\right)= \begin{cases}1 & \left|\omega_{l}\right|<\omega_{1} \\ \frac{\omega_{2}-\left|\omega_{l}\right|}{\omega_{2}-\omega_{1}} & \omega_{1} \leqslant\left|\omega_{l}\right| \leqslant \omega_{2} \\ 0 & \left|\omega_{l}\right|>\omega_{2}\end{cases}
$$

where $\omega_{2}>\omega_{1}>0$.

In Eq. (2)-(4), $z$ is the average deformation amount of bristles. $\omega_{l}$ is load speed. $\sigma_{0}$ is the stiffness coefficient of bristles. $\sigma_{1}$ is the damping coefficient of bristles. $\alpha_{2}$ is the viscous damping coefficient. $F_{c}$ is the coulomb friction coefficient. $F_{s}$ is the static friction coefficient. $\omega_{s}$ is the Stribeck velocity.

When the system is running at low speed, $s(|\omega|)=1, F=\sigma_{0} z+\sigma_{1} \dot{z}+\alpha_{2} \omega$, the friction model is equivalent to the LuGre model. When the system is running at high speed, $s(|\omega|)=0$, $F=F_{c} \operatorname{sgn}(\omega)+\alpha_{2} \omega$, the friction model is equivalent to a static friction model. In the actual LuGre model, parameters will change due to the influence of temperature, lubrication, material wear and other factors. So we use $\lambda$ to reflect the influence of parameter change on the friction torque. Then the load friction torque is as follows.

$$
F=\lambda\left(\sigma_{0} s\left(\left|\omega_{l}\right|\right) z+\sigma_{1} \dot{z}+F_{c} \operatorname{sgn}\left(\omega_{l}\right)\left(1-s\left(\left|\omega_{l}\right|\right)\right)+\alpha_{2} \omega_{l}\right)
$$

So the servo system model can be expressed as follows.

$$
\left\{\begin{array}{l}
\frac{d \theta}{d t}=\omega \\
\frac{d \omega}{d t}=C_{0} u-C_{1} \omega-\lambda C_{2} \omega-\lambda \varphi z-\lambda C_{5} F_{c}-C_{6} T_{l} \\
y=\theta
\end{array}\right.
$$

where,

$$
\begin{aligned}
& C_{0}=\frac{K_{t}}{J}, C_{1}=\frac{b}{J}, C_{2}=\frac{\sigma_{1} s\left(\left|\omega_{l}\right|\right)+\alpha_{2}}{J}, C_{3}=\frac{\sigma_{0} s\left(\left|\omega_{l}\right|\right)}{J}, C_{4}=\frac{\sigma_{0} \sigma_{1} s\left(\left|\omega_{l}\right|\right)}{J}, C_{5}=\frac{\operatorname{sgn}\left(\omega_{l}\right)\left(1-s\left(\left|\omega_{l}\right|\right)\right)}{J}, \\
& C_{6}=\frac{1}{J}, \varphi=C_{3}-\frac{C_{4}|\omega|}{g\left(\omega_{l}\right)} .
\end{aligned}
$$

\section{Backstepping Adaptive Fuzzy Control}

\subsection{Fuzzy Approximation System}

In order to reduce the influence of the friction nonlinearity and the load uncertainty in servo system, we design controller employing adaptive fuzzy approximation system[19] to compensate them. The purpose is to make system can accurately track the given position signal.

We use the following fuzzy inference rules: 
$R^{j}$ :If $x_{1}$ is $F_{1}^{j}$ and $x_{2}$ is $F_{2}^{j} \ldots$ and $x_{n}$ is $F_{n}^{j}$, then $y$ is $G^{j} j=1,2, \cdots, M$

where $x=\left[x_{1}, x_{2}, \cdots, x_{n}\right]^{\mathrm{T}} \in R^{n}$ is the input of fuzzy system, $y \in R$ is the output of fuzzy system, $F_{i}^{j}$ and $G^{j}$ are fuzzy sets.

Using the following fuzzy system:

$$
y=\frac{\sum_{j=1}^{M} \bar{y}^{j}\left(\prod_{i=1}^{n} \mu_{F_{i}^{j}}\left(x_{i}\right)\right)}{\sum_{j=1}^{M} \prod_{i=1}^{n} \mu_{F_{i}^{j}}\left(x_{i}\right)}
$$

where, $\mu_{G^{j}}\left(\bar{y}^{j}\right)$ is fuzzy membership function, $\bar{y}^{j}$ is the maximum point of $\mu_{G^{j}}\left(\bar{y}^{j}\right)$.

Let

$$
q_{j}=\frac{\prod_{i=1}^{n} \mu_{F_{i}}\left(x_{i}\right)}{\sum_{j=1}^{M}\left(\prod_{i=1}^{n} \mu_{F_{i}^{j}}\left(x_{i}\right)\right)}
$$

define $\theta=\left[\bar{y}^{1}, \bar{y}^{2}, \cdots, \bar{y}^{M}\right]^{\mathrm{T}}$ as the adaptive parameter vector of fuzzy system, $q(x)=\left[q_{1}(x), q_{2}(x), \cdots, q_{M}(x)\right]^{\mathrm{T}}$ as fuzzy basis function vector, then Eq. (8) can be expressed as follows.

$$
y(x)=\theta^{\mathrm{T}} q(x)
$$

\subsection{Backstepping Design}

In this paper, we designed an adaptive controller so as to make system output $y$ asymptotically stable tracking the expected output $y_{d}$, namely $\lim _{t \rightarrow \infty}\left|y-y_{d}\right|=0$.

Theorem 1: For any given continuous function $p(x)$ on the compact set $U \in R^{n}$ and arbitrary $\varepsilon>0$, there exists a function $f(x)$ in the form of $(8)$, such that:

$$
\sup _{x \in U}\|f(x)-p(x)\|<\varepsilon
$$

Assumption 1: System parameter $K_{t} / J$ is bounded. There exist $d_{\mathrm{m}}$ and $d_{M}$, such that

$$
0<d_{\mathrm{m}} \leqslant\left|K_{t} / J\right| \leqslant d_{M}
$$

Assumption 2: The first and second derivative of $y_{d}$ exist and are all bounded.

Step 1 Define output error $e_{1}$ as

$$
e_{1}=y-y_{d}=\theta-y_{d}
$$

Its derivative is

$$
\dot{e}_{1}=\dot{\theta}-\dot{y}_{d}=\omega-\dot{y}_{d}=\omega-\omega_{d}
$$

Choose the following control Lyapunov function $V_{1}$

$$
V_{1}=\frac{1}{2} e_{1}^{2}
$$

Its derivative is

$$
\dot{V}_{1}=e_{1} \dot{e}_{1}=e_{1}\left(\omega-\dot{y}_{d}\right)
$$

Let $\omega$ be virtual control variable, define $\alpha_{1}$ as feedback control input, the value of $\alpha_{1}$ is: 


$$
\alpha_{1}=-k_{1} e_{1}+\dot{y}_{d}
$$

where, $k_{1}>0$ is a design parameter, then Eq.(16) can be expressed as follows.

$$
\dot{V}_{1}=e_{1}\left(\omega-\alpha_{1}-k_{1} e_{1}\right)=-k_{1} e_{1}^{2}+e_{1}\left(\omega-\alpha_{1}\right)
$$

Step 2 Define the error between $\omega$ and $\alpha_{1}$ as

$$
e_{2}=\omega-\alpha_{1}
$$

Choose the following augmented control Lyapunov function $V_{2}$

$$
V_{2}=V_{1}+\frac{1}{2} e_{2}^{2}
$$

Its derivative is

$$
\dot{V}_{2}=-k_{1} e_{1}^{2}+e_{2}\left(f^{*}(x)+g(x) u\right)
$$

where,

$$
\begin{gathered}
f^{*}(x)=e_{1}+\left(-b \omega-F-T_{l}\right) / J-\dot{\alpha}_{1} \\
\dot{\alpha}_{1}=\frac{\partial \alpha_{1}}{\partial x_{1}} \dot{x}_{1}+\frac{\partial \alpha_{1}}{\partial y_{d}} \dot{y}_{d}+\frac{\partial \alpha_{1}}{\partial \dot{y}_{d}} \ddot{y}_{d}
\end{gathered}
$$

We can see that $f^{*}(x)$ is an unknown function. For any given accuracy requirement $\varepsilon>0$, there exist a fuzzy logic system, such that

$$
f^{*}=\theta^{\mathrm{T}} q(x)+\delta(x),|\delta(x)|<\varepsilon
$$

where $\delta(x)$ is an approximation error function.

define

$$
\beta=\frac{1}{d_{m}}\|\theta\|^{2}
$$

Define the estimated value of $\beta$ as $\hat{\beta}$ which satisfying the following differential equation.

$$
\dot{\hat{\beta}}=\frac{r}{2 a^{2}} e_{2}^{2} q^{\mathrm{T}} q-\sigma \hat{\beta}
$$

where, $r>0, \sigma>0$ are design parameters.

We choose control law as follows.

$$
u=-\left(k_{2}+0.5\right) e_{2}-\frac{1}{2 a^{2}} e_{2} \hat{\beta} q^{\mathrm{T}} q
$$

where, $k_{2}>0, a>0$ are design parameters.

then we can get

$$
\begin{gathered}
e_{2} f^{*}=e_{2} \theta^{\mathrm{T}} q(x)+e_{2} \delta(x) \leqslant \frac{1}{2 a^{2}} e_{2}^{2} q^{\mathrm{T}} q\|\theta\|^{2}+\frac{a^{2}}{2} \frac{\theta^{\mathrm{T}} \theta}{\|\theta\|^{2}}+\frac{1}{2 d_{m}} \varepsilon^{2} \\
e_{2} g(x) u \leqslant-\left(k_{2}+0.5\right) d_{m} e_{2}^{2}-\frac{d_{m}}{2 a^{2}} e_{2}^{2} \hat{\beta} q^{\mathrm{T}} q
\end{gathered}
$$

substitute Eqs.(28),(29) into Eqs.(21), we can get

$$
\dot{V}_{2} \leqslant-k_{1} e_{1}^{2}-k_{2} d_{m} e_{2}^{2}+\frac{1}{2} a^{2}+\frac{1}{2 d_{m}} \varepsilon^{2}+\frac{d_{m}}{2 a^{2}} e_{2}^{2} q^{T} q \tilde{\beta}
$$

where $\tilde{\beta}=\beta-\hat{\beta}$

\subsection{Stability Analysis}

We choose the augmented control Lyapunov function as follows. 


$$
V_{3}=V_{2}+\frac{d_{m}}{2 r} \tilde{\beta}^{2}
$$

Its derivative is

$$
\begin{gathered}
\dot{V}_{3} \leqslant-k_{1} e_{1}^{2}-k_{2} d_{m} e_{2}^{2}+\frac{1}{2} a^{2}+\frac{1}{2 d_{m}} \varepsilon^{2}+\frac{d_{m}}{2 a^{2}} e_{2}^{2} q^{\mathrm{T}} q \tilde{\beta}-\frac{d_{m}}{r} \tilde{\beta} \dot{\hat{\beta}}=-k_{1} e_{1}^{2}-k_{2} d_{m} e_{2}^{2}+\frac{1}{2} a^{2}+\frac{1}{2 d_{m}} \varepsilon^{2} \\
+\frac{d_{m}}{r} \tilde{\beta}\left(\frac{r}{2 a^{2}} e_{2}^{2} q^{\mathrm{T}} q-\dot{\hat{\beta}}\right)=-k_{1} e_{1}^{2}-k_{2} d_{m} e_{2}^{2}+\frac{1}{2} a^{2}+\frac{1}{2 d_{m}} \varepsilon^{2}+\frac{\sigma d_{m}}{r} \tilde{\beta} \hat{\beta}
\end{gathered}
$$

notice that

$$
\tilde{\beta} \hat{\beta}=\tilde{\beta}(\beta-\tilde{\beta}) \leqslant \frac{1}{2} \beta^{2}-\frac{1}{2} \tilde{\beta}^{2}
$$

So Eq. (32) can be expressed as follows.

$$
\dot{V}_{3} \leqslant-k_{1} e_{1}^{2}-k_{2} d_{m} e_{2}^{2}+\frac{1}{2} a^{2}+\frac{1}{2 d_{m}} \varepsilon^{2}+\frac{\sigma d_{m}}{2 r} \beta^{2}-\frac{\sigma d_{m}}{2 r} \tilde{\beta}^{2} \leqslant-m_{0} V_{3}+n_{0}
$$

where, $m_{0}=\min \left\{2 k_{1}, 2 d_{m} k_{2}, \sigma\right\}, n_{0}=\frac{1}{2} a^{2}+\frac{1}{2 d_{m}} \varepsilon^{2}+\frac{\sigma d_{m}}{2 r} \beta^{2}$

Calculating the integral on both sides of eq.(34), we have:

According to eqs.(31),(35), we have:

$$
V_{3}(t) \leqslant e^{-m_{0} t} V_{3}(0)+\frac{n_{0}}{m_{0}}\left(1-e^{-m_{0} t}\right)
$$

$$
e_{1}^{2} \leqslant 2 V_{3}(t), \lim _{t \rightarrow \infty} e_{1}(t) \leqslant \sqrt{\frac{2 n_{0}}{m_{0}}}
$$

Because $k_{1}, k_{2}, r, \sigma, a$ are all design parameters, we can appropriately choose them to ensure that $n_{0} / m_{0}$ is small sufficiently, which can ensure that the error $e_{1}$ converges to a sufficient small neighborhood of the origin according to the exponential law.

\section{Simulation Experiment}

The following simulation graphics are the comparison charts of backstepping adaptive fuzzy control (BAFC) and the conventional PID control.

The parameters of servo system model are as follows: $J=0.8 \mathrm{~kg} \cdot \mathrm{m}^{2}, K_{t}=1, b=0.5 \mathrm{~N} \cdot \mathrm{m} \cdot \mathrm{s} / \mathrm{rad}$, $i=15$. The parameters of LuGre friction model are as follows: $\sigma_{0}=9500 \mathrm{~N} \cdot \mathrm{m}, \sigma_{1}=25 \mathrm{~N} \cdot \mathrm{m}$, $\sigma_{2}=0.3 \mathrm{~N} \cdot \mathrm{m} \cdot \mathrm{s} / \mathrm{rad}, F_{c}=0.5 \mathrm{~N} \cdot \mathrm{m}, F_{s}=0.45 \mathrm{~N} \cdot \mathrm{m}, \omega_{s}=0.01 \mathrm{rad} / \mathrm{s}$. The initial state is: $\theta=0$, $\omega=0$. The design parameters are: $k_{1}=90, k_{2}=80, a=0.3, r=6.2, \sigma=0.6$. The fuzzy membership functions are as follows.

$$
\begin{aligned}
& \mu_{F_{i}^{1}}\left(x_{i}\right)=1 /\left(1+\exp \left(-6\left(x_{i}-6\right)\right)\right), \mu_{F_{i}^{2}}\left(x_{i}\right)=\exp \left(-0.5\left(x_{i}-4\right)^{2} / 2\right), \mu_{F_{i}^{3}}\left(x_{i}\right)=\exp \left(-0.5\left(x_{i}-2\right)^{2} / 2\right) \\
& \mu_{F_{i}^{4}}\left(x_{i}\right)=\exp \left(-0.5\left(x_{i}-0\right)^{2} / 2\right), \mu_{F_{i}^{5}}\left(x_{i}\right)=\exp \left(-0.5\left(x_{i}+2\right)^{2} / 2\right), \mu_{F_{i}^{6}}\left(x_{i}\right)=\exp \left(-0.5\left(x_{i}+4\right)^{2} / 2\right) \\
& \mu_{F_{i}^{7}}\left(x_{i}\right)=1 /\left(1+\exp \left(6\left(x_{i}+6\right)\right)\right)
\end{aligned}
$$

\subsection{System Response Analysis}

Fig. 2(a) is the system position tracking curve for sinusoidal signal input $y_{d}=\sin (1.2 \pi t)$ by using PID control and BAFC, respectively. Fig. 2(b) is the error curve between $y$ and $y_{d}$ for sinusoidal 
signal input. Fig. 3(a) is the system velocity tracking curve for sinusoidal signal input $y_{d}=\sin (1.2 \pi t)$ by using PID control and BAFC, respectively. Fig. 3(b) is the error curve between $\omega$ and $\omega_{d}$ for sinusoidal signal input. Fig. 4 is the controller output curve by using PID control and BAFC, respectively. From Fig. 2 and Fig. 3, we can see that position tracking exist flat top phenomenon and velocity tracking exists deadzone phenomenon when using PID control. But there are no flat top phenomenon and deadzone phenomenon exist when using BAFC. So the system has better tracking performance and smaller tracking error when using BAFC.

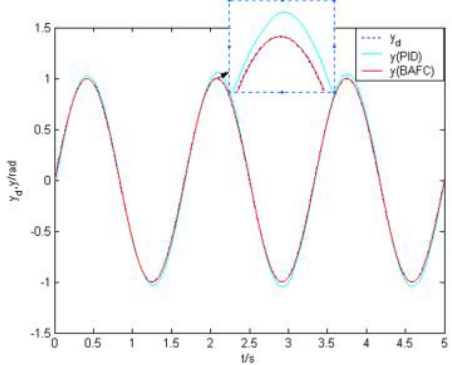

(a) System position tracking for sinusoidal signal

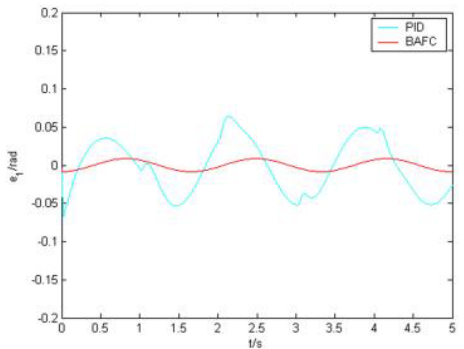

(b) Position tracking error for sinusoidal signal Figure 2. System position tracking and tracking error for sinusoidal signal input.

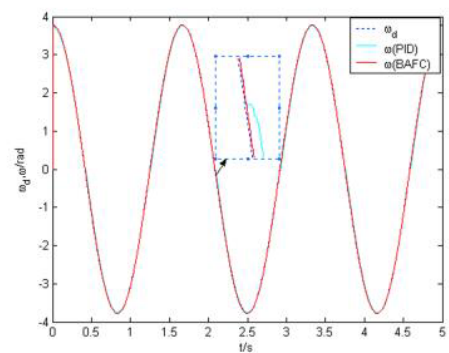

(a) System velocity tracking for sinusoidal signal

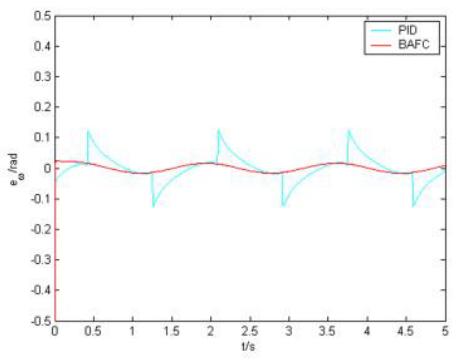

(b) Velocity tracking error for sinusoidal signal Figure 3. System velocity tracking and tracking error for sinusoidal signal input.

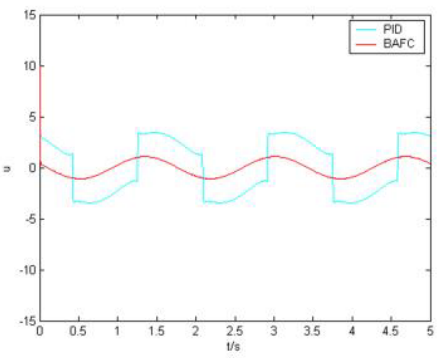

Figure 4. System controller output.

\subsection{System Robustness Analysis}

In Fig. $5,10 \mathrm{~N} \cdot \mathrm{m}$ disturbance signal is applied to system at $2 \mathrm{~s}$, the system not only has a larger fluctuation at $2 \mathrm{~s}$ but also the tracking performance become worse after $2 \mathrm{~s}$ when using PID control. But the system tracking performance don't become worse after $2 \mathrm{~s}$, only has a smaller fluctuation at $2 \mathrm{~s}$ when using BAFC. Thus BAFC has better robustness than PID control. 


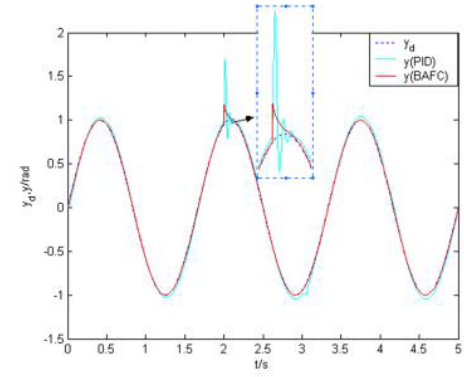

(a) System sinusoidal position tracking containing disturbance

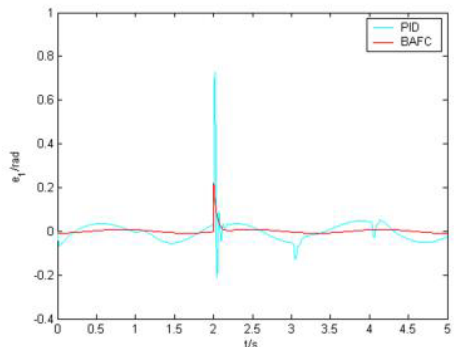

(b) Sinusoidal position tracking error containing disturbance

Figure 5. System position tracking and tracking error for sinusoidal signal when disturbance exists.

\section{Conclusion}

In this paper, we designed the improved LuGre friction model-based backstepping adaptive fuzzy control of servo system. The proposed control strategy has the characteristics of higher tracking accuracy, better robustness and adaptability than conventional PID control. But the system also exists backlash nonlinearity, we don't consider the coexistence of backlash and friction nonlinearlity in the system. It will be our future work considering the coexistence of them.

\section{Acknowledgment}

This work was supported by National Natural Science Foundation of China(No.61074023), Nature Science Foundation in Anhui Province of China(No.1508085MF130), Natural Science Research Key Project of Universities in Anhui Province of China (No.KJ2015A297), the Engineering Technology Research Center of Optoelectronic Appliance in Anhui Province of China, Sichuan Institute of Aerospace System Engineering in Sichuan Province of China.

The authors confirm that this article content has no conflicts of interest.

\section{References}

1. J. Zhou, B. Duan, J. Huang, Effect and compensation for servo systems using LuGre friction model, Control Theor. App. 25(6) (2008) 990-994.

2. S. K. Shome, M. Prakash, S. Pradhan, Robust dahl model based Sliding Mode Control for micro/nano positioning applications, 11th IEEE India Conference: Emerging Trends and Innovation in Technology, February 3, 2015.

3. H. Xie, Z. Liu, H. Yang, Advancing control for shield tunneling machine by Backstepping design with lugre friction model, Math. Probl. Eng. ten pages, 2014.

4. I. Redaelli, C. di Prisco, D. Vescovi, A visco-elasto-plastic model for granular materials under simple shear conditions, Int. J. Numer. Anal. Methods Geomech. 40(1) (2016) 80-104.

5. V. Lampaert, J. Swevers, F. Al-Bender, Modification of the Leuven integrated friction model structure, IEEE T. Autom. Control 47(4) (2002) 683-687.

6. I. Nilkhamhang, A. Sano, Robust adaptive friction compensation using linearly-parameterized GMS model, 9th European Control Conference, 2015, 1314-1319.

7. W. Tan, X. Li, H. Xiang, Research on compensation of torque ripple in servo system, J. Mech. Eng. 47(12) (2011) 1-6.

8. Y. Wang, Z. He, Friction compensation for servo systems, Electric Mach. Control 17(8) (2013) 107-112. 
9. S. Yang, M. Zeng, New repetitive adaptive friction compensation scheme in high-precise servo system, J. Southeast U. 36(SUPPL) (2006) 74-48.

10. Y. Wu, D. Ma, J. Yao, Application of adaptive robust control in mechatronic servo system based on modified LuGre model, J. Mech. Eng. 50(22) (2014) 207-212.

11. Q. Wu, X. Wang, F. Du, Torque transmission characteristics and friction compensation for a tendon-sheath actuation system in pull-pull configuration, J. Mech. Eng. 51(5) (2015) 22-29.

12. B. Feng, X. S. Mei, J. Yang, Adaptive configuration method of friction compensation pulse characteristic parameters, J. Shanghai Jiaotong U. 58(5) (2014) 713-718.

13. M. Ruderman, Tracking control of motor drives using feedforward friction observer, IEEE T. Ind. Electron. 61(7) (2014) 3727-3735.

14. T. S. Aung, R. Kikuuwe, M. Yamamoto, Friction compensation of geared actuators with high presliding stiffness, J. Dyn. Syst. Meas. Control, T. ASME 137(1) (2015) 8 pages.

15. L. X. Wang, J. M. Mendel, Fuzzy basis functions, universal approximation, and orthogonal leastsquares learning, IEEE T. Neural Net. 3(5) (1992) 807-814.

16. J. P. Yu, B. Chen, H. S. Yu, Adaptive fuzzy backstepping position tracking control for permanent magnet synchronous motor, Control and Decision, 25(10) (2010) 1547-1551.

17. H. Chaoui, P. Sicard, Adaptive fuzzy logic control of permanent magnet synchronous machines with nonlinear friction, IEEE T. Ind. Electron. 59(2) (2010) 1123-1133.

18. X. Guo, Q. Wang, G. Li, Adaptive fuzzy control for permanent magnet spherical motor based on friction compensation, P. Chin. Soc. Electrical Eng. 31(15) (2011) 75-81.

19. Y. F. Wang, T. Y. Chai, Adaptive fuzzy control method for dynamic friction compensation, P. CSEE, 25(2) (2005) 139-143.

20. Y. F. Wang, D. H. Wang, T. Y. Chai, Extraction and adaptation of fuzzy rules for friction modeling and control compensation, IEEE t. fuzzy syst. 19(4) (2011) 682-693.

21. L. Lu, B. Yao, Q. Wang, Adaptive robust control of linear motors with dynamic friction compensation using modified LuGre model, Automatica, 45(12) (2009) 2890-2896.

22. Q. Liang, J. Zhang, Y. Wang, Adaptive backstepping friction compensation control based on modified LuGre model, Small Special Electrical Mach. (11) (2011) 67-69.

23. D. K. Wang, Backstepping based adaptive fuzzy control and its application, J. Shengyang Normal U. 31(2) (2013) 192-195.

24. H. B. Zhao, X. H. Zhou, Backstepping adaptive control of dual-motor driving servo system, Control Theor. Appl. 28(5) (2011) 745-751. 